\title{
Scanning Electron Microscopic Study of Cylindrical Structures Observed in Human Dentinal Tubules: The Controversy Continues
}

\author{
G.D. Hack*, M. Salnik**, R. Hsia***
}

* Department of Endodontics, Prosthodontics, and Operative Dentistry, University of Maryland Dental School, Baltimore, MD 21201-1586

** Dental Student, University of Maryland Dental School, Baltimore, MD 21201-1586

*** Electron Microscopy Core Facility, University of Maryland, Baltimore, Baltimore, MD 21201-1586

Debate still exists as to the exact nature of cylindrical structures (CS) occasionally observed within the dentinal tubules of human teeth. SEM is the most often utilized method of examining human dentin [1]. The classic view maintains that the dentinal tubules are occupied by cell processes throughout their entire length. While some investigations have described the cell processes as being restricted to the inner aspect of dentin, others have described these structures, using cryo-EM, throughout the entire length of the tubules. Still other studies, using a combination of both SEM and TEM, have identified these structures as lamina limitans, an extracellular organic matrix which in certain conditions may appear detached from the internal wall of the tubule, and simulate the appearance of a cellular process [2]. Recently, these CS have been described as the hyphal branches of yeast cells (C. albicans) which have infected the tooth structure [3] .

The aim of the present study was to investigate these CS using SEM with various specimen preparation techniques. Acid-etched dentin specimens were evaluated by means of Quanta 200 SEM. This examination evidenced CS emanating from some dentinal tubules in specific areas of the dentin surface, averaging 1.5 microns in diameter. The CS were smaller in diameter then the tubules from which they emerged, and the majority of the CS appeared to be hollow (Fig. 1). Other dentin samples revealed CS that appeared to be more solid, and one tubule contained two distinct CS (Fig. 2). Still another dentin specimens revealed a comparatively thin, hollow CS emerging from a single dentinal tubule (Fig. 3).

Our observations raise additinal questions in this continuing debate. The diameter of the CS observed in this study are not consistent with that of cellular extensions, which are reported to be 0.5 microns in diameter. The size of the observed CS were more consistent with that of the lining of dentinal tubules or lamina limitans. Interestingly, our observation of two CS within one tubule challenges even this hypothesis. Future studies are planned utilizing FIB, TEM, and cryoEM to aid in elucidating the true nature of these controversial structures.

[1] B. Jodlowska-Jedrych et al., Microsc. Microanal. 14 (suppl. 2) (2008) 1456CD.

[2] S. Muylle et al., Arch. Oral Biol. 29 (2000) 641-646.

[3] T.M.T. Waltimo et al., Crit. Rev. Oral Biol. Med. 14 (2003) 128-137. 

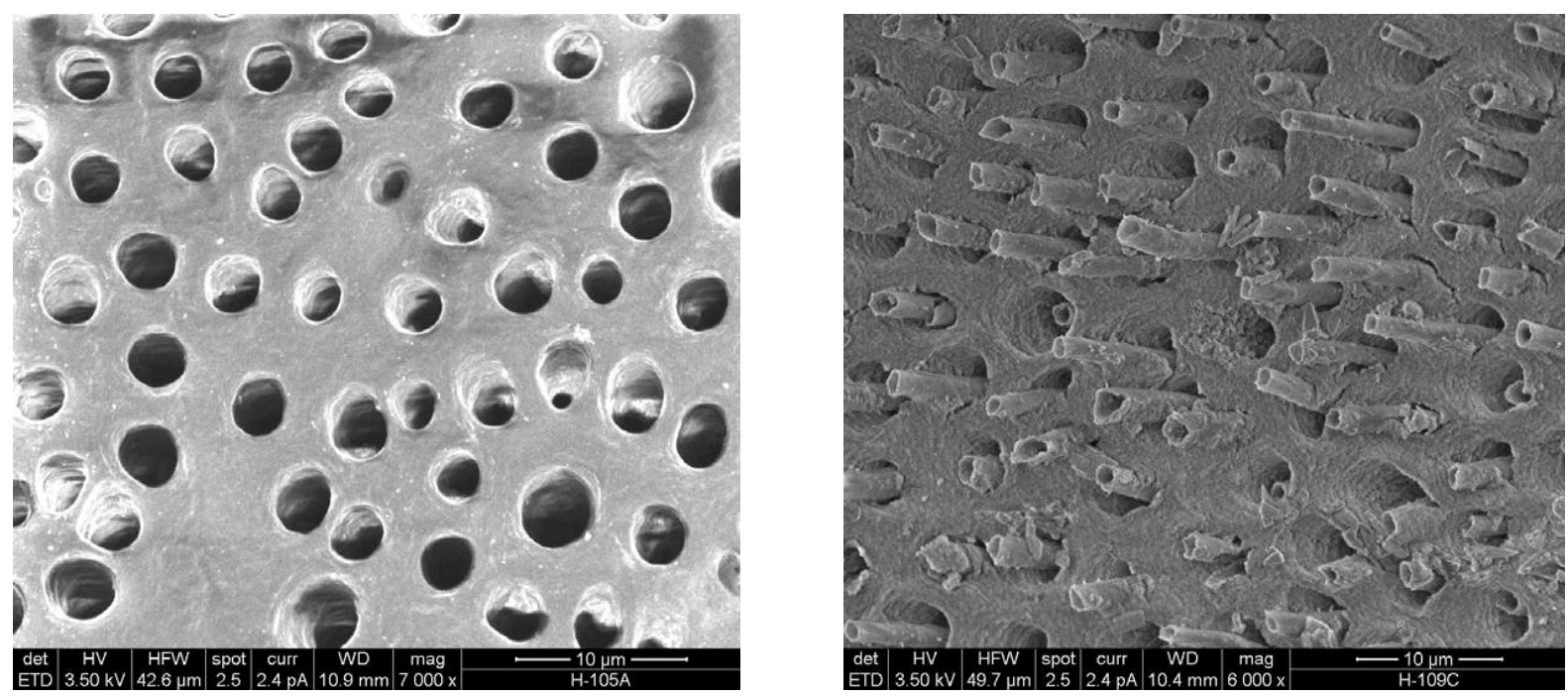

FIG. 1. Etched dentin surface observed in cross-section. The majority of the dentin surface evidenced empty dentinal tubules (left). Area of dentin evidencing a group of dentinal tubules with cylindrical structures emanating from within the tubules (right).

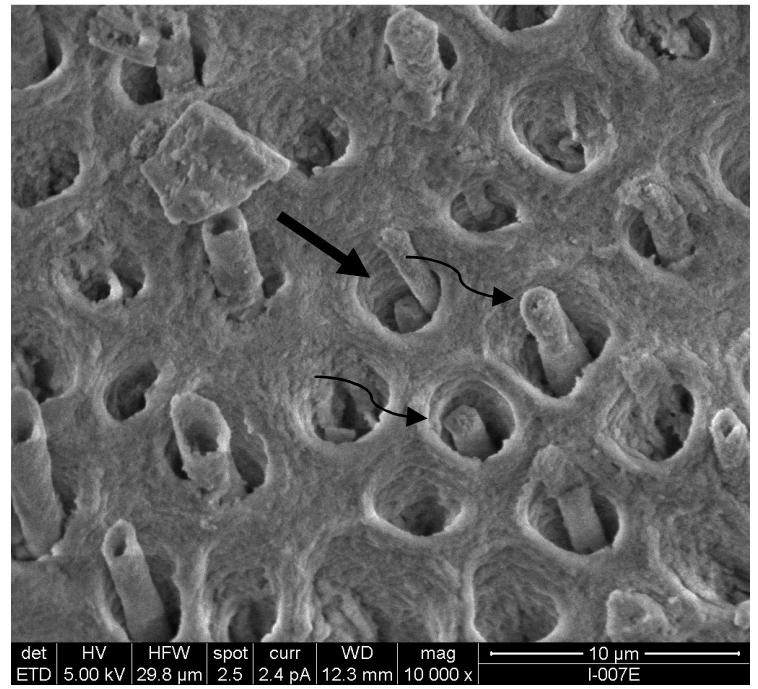

FIG. 2. Note solid cylindrical structures (thin arrows), and two structures existing in a single tubule (arrow).

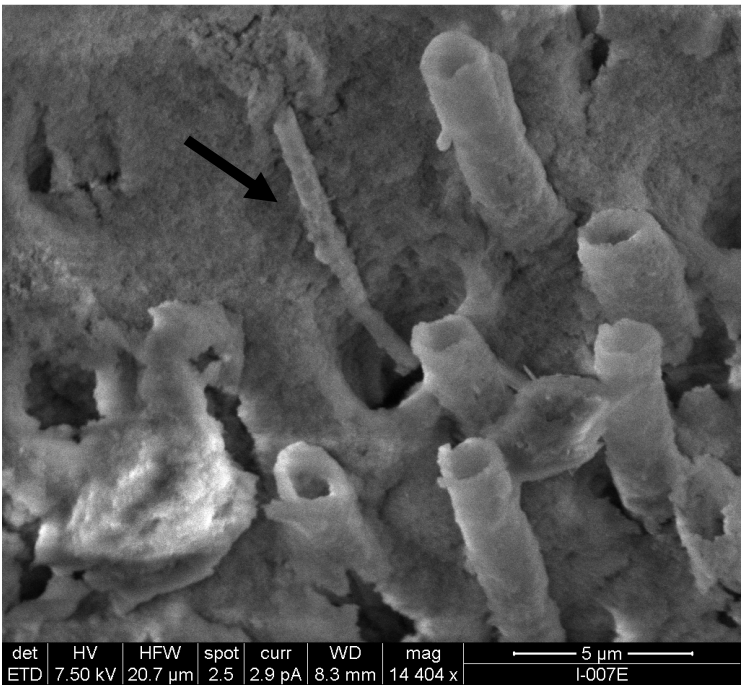

FIG. 3. Note thin cylindrical structure emanating from the dental tubule (arrow). 